\title{
Effects of Autologous Platelet-Rich Plasma on Endometrium Thickness and Pregnancy Rates During Intrauterine Insemination
}

\author{
Mehmet Sipahi ${ }^{1}$ \\ ${ }^{1}$ Department of Obstetrics and Gynecology, Faculty of Medicine, Giresun University, Giresun, Turkey
}

Received: 25 March 2019, Accepted 14 May 2019, Published online: 28 August 2019

(C) Ordu University Institute of Health Sciences, Turkey, 2019

\begin{abstract}
Objective: Evaluation of the effect of platelet-rich plasma (PRP) on the endometrium and pregnancy outcomes in patients undergoing insemination due to unexplained infertility.

Methods: 24 patients who were admitted to the clinic due to unexplained infertility, analyzed retrospectively between March 2018 and October 2018. Gonadotropin induction was initiated on day 3 of the cycle for follicular growth. Human chorionic gonadotropin (hCG) was applied for ovulation induction at the point that at least 1 follicle that is over $16 \mathrm{~mm}$ was detected by transvaginal ultrasound. $17.5 \mathrm{ml}$ of blood from the patient's venous system was drawn for the preparation of the PRP which includes 4-5 times more platelets than regular blood. PRP was administered to 12 patients (Group 1) on the hCG day, while hCG was solely administered to the other group (Group 2) and both groups were inseminated 36 hours later.

Results: The demographic properties of all patients were determined as follows: mean age; 29.13 years old $( \pm 3.4)$, mean infertility period; 1.96 years $( \pm 1.08)$, mean ovulation induction period; 7.92 days $( \pm 1.76)$, mean antral follicle count; $14.54( \pm 6.56)$, mean dominant follicle count; $2.04( \pm 0.75)$. Although there was no significant difference between the groups in terms of clinical pregnancy (3/12 vs $2 / 12$, p: 0.623$)$, the change in endometrial thickness was significantly higher in the PRP administered group $(1.95 \mathrm{~mm}$ vs 0.44 $\mathrm{mm}, \mathrm{p}<0.001)$.
\end{abstract}

Conclusion: PRP application before the insemination seems promising for the preparation of the endometrium in patients having an inadequate endometrial thickness or in patients experiencing recurrent implantation failure.

Key words: Intrauterine insemination, endometrial thickness, autologous platelet-rich plasma, infertility, pregnancy rate

Suggested Citation: Sipahi M. Advanced Maternal Age and Adverse Perinatal Outcomes - One Decade Analysis. Middle Black Sea Journal of Health Science, 2019; 5(1): 63-66

Address for correspondence/reprints:

Mehmet Sipahi

Telephone number: +90 (454) 2161108

E-mail: mehmetsipahi@ hotmail.com

DOI: $10.19127 / \mathrm{mbsjohs.544429}$

\section{Introduction}

Unexplained infertility; is the failure to achieve pregnancy despite having unprotected regular sexual intercourse for the period of 12 months (which is the period of 6 months for the women that are above the age of 35) without a definable cause according to American Society for Reproductive Medicine (ASRM, 2008). 15\% of the all infertility reasons are included in this group (Collins and Crosignani, 1992). Factors such as folliculogenesis, luteal phase defect, cervical factor and implantation failure are considered as possible causes. The rate 
of spontaneous pregnancy in these patients without a treatment is $1 \%$ to $3 \%$ per month (Evers, 2002; van Eekelen et al., 2018). It is expected that the treatment option will increase the pregnancy rate above this level. Therefore, to eliminate infertility reasons including cervical factor, intrauterine insemination is a commonly used treatment modality for the unexplained infertility cases.

Recurrent implantation failure is also a frequently discussed topic at in vitro fertilization. Embryo quality, endometrial receptivity, and immunological factors have been subject to research in etiology and, although there have been different treatment options such as blastocyst transfer, pre-implantation genetic screening (PGS), assisted hatching, different culture mediums, endometrial scratching and immunotherapy, no consensus has been achieved yet (Margalioth et al., 2006; Green et al., 2015; Choi et al., 2016). Recently, intrauterine administration of PRP has been described as to accelerate endometrial growth and improve implantation achievement (Chang et al., 2015). PRP is an autologous serum, which contains various growth factors and cytokines such as fibroblast growth factor, platelet-derived growth factor, vascular endothelial growth factor, transforming growth factor, insulin-like growth factor I-II, connective tissue growth factor and IL-8 (Chang et al., 2015; Nazari et al., 2016) . Although PRP has found widespread use for tissue healing, especially in areas such as ophthalmology, orthopedics and plastic surgery, there are very limited number of existing studies in the field of infertility. (Dhillon et al., 2012; Chang et al., 2015; Ronci et al., 2015; Garcia-Velasco et al., 2016; Sfakianoudis et al., 2018).

The aim of our study was to investigate the effect of PRP on endometrium and pregnancy outcomes in patients undergoing intrauterine insemination IUI due to unexplained infertility

\section{Methods}

24 patients, who were admitted to Giresun University Gynecology and Obstetrics Clinic due to unexplained infertility, were analyzed retrospectively since March 2018 to October 2018. PRP was administered to 12 patients (Group 1) on the hCG day, while hCG was solely administered to the other group (Group 2) and both groups were inseminated 36 hours later. Being exposed to clomiphene citrate treatment at least twice, existence of tubal patency, and being under the age of 35 were defined as inclusion criteria, while the exclusion criteria was determines as existence of a structural or anatomical condition that could prevent reproduction, any sort of systemic disease, patients with BMI over 30 , having total progressive sperm concentration (TPSC) under 10 million and having an endometrium under $7 \mathrm{~mm}$ at hCG day.

Gonadotropin induction was initiated on day 3 of the cycle for follicular growth. Recombinant hCG (Ovitrelle $250 \mu \mathrm{g}$, Merck) was applied for ovulation induction at the point that at least 1 follicle that is over $16 \mathrm{~mm}$ was detected by transvaginal ultrasound. Endometrium thicknesses on hCG and insemination day were also noted.

$17.5 \mathrm{ml}$ of blood from the patient's venous system was drawn for the preparation of the PRP. Then, centrifugation at $1200 \mathrm{rpm}$ for 12 minutes was applied to eliminate red blood cells and remaining plasma was centrifugated at $3000 \mathrm{rpm}$ for 7 minutes to achieve PRP having 4-5 times more platelets when compared to regular blood (Nazari et al., 2016). hCG was administered after $0.5 \mathrm{~mL}$ PRP was given to endometrial cavity with a soft catheter (Medbar, Turkey) without touching to the fundus of uterus and (IUI) was applied 36 hours later. Microfluidic sperm sorting chip was used for sperm preparation in all patients (Fertil Plus Koek Ltd., Turkey).

Ethics committee approval was received from Giresun University Ethical Committee (KAEK2017/32) and all patients have signed their volunteer consent forms as being informed.

\section{Results}

The demographic properties of all patients that underwent IUI due to unexplained infertility were determined as following: mean age; 29.13 years old (Standart Deviation: [SD]: \pm 3.4 ), mean infertility period; 1.96 year (SD: \pm 1.08 ), mean follicle stimulating hormone (FSH) level; $6.38 \mathrm{mIU} / \mathrm{mL}$ (SD: \pm 1.55 ), mean estradiol (E2) level; 47.75 $\mathrm{pg} / \mathrm{mL}(\mathrm{SD}: \pm 31.26)$, mean ovulation induction period; 7.92 days (SD: \pm 1.76 ), mean gonadotrophin dose; 775,52 IU (SD: \pm 198.85 ), mean antral follicle count; 14.54 (SD: \pm 6.56 ), mean dominant follicle count; 2.04 (SD: \pm 0.75 ). The distribution of demographic properties and clinical findings of groups are summarized below in Table 1 .

Although there was no significant difference between the two groups in terms of clinical pregnancy ( $p: 0.623$ ), the change in endometrial thickness was significantly higher in the PRP implemented group $(1.95 \mathrm{~mm}$ vs $0.44 \mathrm{~mm}, \mathrm{p}$ $<0.001)$. 
Table 1. Demographic properties and findings of groups

\begin{tabular}{|c|c|c|}
\hline & Group $1( \pm \mathrm{SD})$ & Group $2( \pm \mathrm{SD})$ \\
\hline Age (year) & $29.58(3.6)$ & $28.67(3.2)$ \\
\hline Infertility period (year) & $2.33(1.2)$ & $1.58(0.7)$ \\
\hline FSH 3. day $(\mathrm{mIU} / \mathrm{mL})$ & $6.50(1.5)$ & $6.25(1.6)$ \\
\hline TPSC & $94.10^{6}$ & $124.10^{6}$ \\
\hline Induction period (day) & $8.25(2.1)$ & $7.58(1.2)$ \\
\hline $\operatorname{AFC}(\mathrm{N})$ & $13.83(3.5)$ & $15.25(8.7)$ \\
\hline $\begin{array}{l}\text { End thickness at hCG } \\
\text { day }(\mathrm{mm})\end{array}$ & $9.15(1.6)$ & $10.49(2.2)$ \\
\hline $\begin{array}{l}\text { End thickness at IUI } \\
\text { day }(\mathrm{mm})\end{array}$ & $11.10(1.5)$ & $10.93(2.3)$ \\
\hline $\begin{array}{l}\text { Change of end } \\
\text { thickness }(\mathrm{mm}) *\end{array}$ & $1.95(0.4)$ & $0.44(0.1)$ \\
\hline $\begin{array}{l}\text { Clinical pregnancy }(\%) \\
-[\mathrm{N}] \mathrm{I}\end{array}$ & $25(0.4)-[3 / 12]$ & $17(0.3)-[2 / 12]$ \\
\hline
\end{tabular}

\section{Discussion}

IUI is a frequently used treatment modality for couples who are admitted for unexplained infertility. Although it has a low success rate comparing to in vitro fertilization, IUI is an economical and less invasive method. Since the cause of infertility cannot be fully established, it is still arguable as when the treatment should be initiated and how to proceed. There was no significant difference for expectant management between the group that started the treatment with IUI as delayed for 6 months and the group that initiated treatment with IUI immediately in a study that compared 253 patients to find out the effect of pre-treatment expectant period. (RR: $0.99,95 \% \mathrm{CI}$ : 0.85-1.1) (Custers et al., 2012).

Similarly, 14 studies and 1867 women were examined in a meta-analysis comparing IUI, timely intercourse and expectant management in terms of live birth rates and multiple pregnancies in natural and stimulated cycles. The only significant increase in live birth rates were detected when stimulated IUI cycles $(25 \%)$ were compared to IUI $(9-21 \%)$ in the natural cycles (OR: 0.48, 95\% CI:0.29-0.82; 4 RCT, n:396, I2:0) (Veltman-Verhulst et al., 2016).

This study has designed IUI treatment for 24 patients, who had been failed for pregnancy with clomiphene citrate at least 2 times without tubal factor with unexplained infertility. Assuming that it could increase endometrial growth and receptivity, the study also applied PRP to the group 1. Although there was no significant difference between the two groups in terms of pregnancy, the increase in endometrial thickness was higher in the PRP implemented group $(0.44 \mathrm{~mm}$ vs $1.95 \mathrm{~mm}$; $\mathrm{p}<0.001)$.

During the period, the endometrium is getting ready for implantation of the embryo between the 19th and the 23rd days of the menstrual cycle, which is known as implantation window at the midsecretory phase (Nazari et al., 2016). Cytokines, growth factors, prostaglandins and adhesion molecules increase at this stage in the endometrium for embryo implantation and development. Among the possible causes of unexplained infertility, PRP was tried at in vitro fertilization in order to treat implantation failure, immunological factors and thin endometrium (Chang et al., 2015; Nazari et al., 2016; Zadehmodarres et al., 2017). Numerous cytokines and growth factors in the PRP may increase the proliferation and receptivity of the endometrium. In a study, PRP was applied to 5 patients that had not received embryo transfer because the endometrial thickness remained below $7 \mathrm{~mm}$ despite estrogen treatment was administered in the previous IVF trial. The expected increase in endometrium thickness was achieved after 48-72 hours of PRP administration. Then, 4 patients received blastocysts and 1 patient received 3rd-day embryo and all had clinical pregnancies (Chang et al., 2015).

In a similar study, 10 patients, whose frozen embryo transfer was canceled due to insufficient endometrial growth in the previous cycle, were examined. The endometrial thickness was enhanced above $7 \mathrm{~mm}$ and embryo transfer was performed in all patients after PRP was given twice as the second dose was applied 48 hours later than the first one. As a result, pregnancy was detected in 5 patients but one resulted in premature abortion (Zadehmodarres et al., 2017). Consequently, PRP can be used for maturation of the thin endometrium according to these studies.

In a different study, 20 patients, who underwent IVF because of recurrent implantation failure, were given PRP 48 hours prior to transfer in frozenthawed cycles. Although the total number of pregnancies were 18 , clinical pregnancy number was 16 because of an early abortion and a molar pregnancy (Nazari et al., 2016). In another study conducted in patients with recurrent implantation failure, it was found that these patients had fewer growth factors in endometrium comparing to fertile patients (Sak et al., 2013). Thus, increased cytokines and growth factors with PRP may have a critical role in the implantation of the embryo.

Since there is a limited number of studies on the use of PRP in the field of infertility, having a small number of patients included in the study was a con, while the first application of PRP for IUI and the 
use of the microfluidic sperm sorting chips were the significant pros of this study

\section{Conclusion}

PRP application before the insemination seems promising for the preparation of the endometrium in patients with inadequate endometrial thickness or in patients with recurrent implantation failure. It is believed that more studies involving more patients are needed to make the use of autologous PRP more reliable in clinical practice.

Ethics Committee Approval: Ethics committee approval was received for this study from Clinical Research Ethics Committee of Giresun University. KAEK-2017-32.

Peer-review: Externally peer-reviewed.

Author Contributions: Concept- M.S.; DesignM.S.; Supervision- M.S.; Funding- M.S.; MaterialsM.S.; Data Collection/Data Process- M.S.; Analyze or Comment- M.S.; Literature Scanning- M.S.; Writer of Paper- M.S.; Critical Review- M.S.

Conflict of Interest: No conflict of interest was declared by the author.

Financial Disclosure: The author declared that this study hasn't received no financial support.

\section{References}

Chang Y, Li J, Chen Y, Wei L, Yang X, Shi Y, et al. Autologous platelet-rich plasma promotes endometrial growth and improves pregnancy outcome during in vitro fertilization. Int J Clin Exp Med. 2015;8:1286-90.

Choi Y, Kim HR, Lim EJ, Park M, Yoon JA, Kim YS, et al. Integrative Analyses of Uterine Transcriptome and MicroRNAome Reveal Compromised LIF-STAT3 Signaling and Progesterone Response in the Endometrium of Patients with Recurrent/Repeated Implantation Failure (RIF). PLoS One. 2016;11:e0157696.

Collins JA, Crosignani PG. Unexplained infertility: a review of diagnosis, prognosis, treatment efficacy and management. Int $\mathbf{J}$ Gynaecol Obstet. 1992;39:267-75.

Custers IM, van Rumste MM, van der Steeg JW, van Wely M, Hompes PG, Bossuyt P, et al. Long-term outcome in couples with unexplained subfertility and an intermediate prognosis initially randomized between expectant management and immediate treatment. Hum Reprod. 2012;27:444-50.

Dhillon RS, Schwarz EM, Maloney MD. Platelet-rich plasma therapy - future or trend?. Arthritis Res Ther. 2012;14:219.
Evers JL. Female subfertility. Lancet. 2002;360:1519.

Garcia-Velasco JA, Acevedo B, Alvarez C, Alvarez M, Bellver J, Fontes J, et al . Strategies to manage refractory endometrium: state of the art in 2016. Reprod Biomed Online. 2016;32:474-89.

Green CJ, Fraser ST, Day ML. Insulin-like growth factor 1 increases apical fibronectin in blastocysts to increase blastocyst attachment to endometrial epithelial cells in vitro. Hum Reprod. 2015;30:284-98.

Margalioth EJ, Ben-Chetrit A, Gal M, Eldar-Geva T. Investigation and treatment of repeated implantation failure following IVF-ET. Hum Reprod. 2006;21:3036-43.

Nazari L, Salehpour S, Hoseini S, Zadehmodarres S, Ajori L. Effects of autologous platelet-rich plasma on implantation and pregnancy in repeated implantation failure: A pilot study. Int J Reprod Biomed. 2016;14:625-8.

Practice Committee of American Society for Reproductive Medicine (ASRM). Definitions of infertility and recurrent pregnancy loss. Fertil Steril. 2008;90:S60

Ronci C, Ferraro AS, Lanti A, Missiroli F, Sinopoli S, Del Proposto G, et al. Platelet-rich plasma as treatment for persistent ocular epithelial defects. Transfus Apher Sci. 2015;52:300-4.

Sak ME, Gul T, Evsen MS, Soydinc HE, Sak S, Ozler A, et al. Fibroblast growth factor-1 expression in the endometrium of patients with repeated implantation failure after in vitro fertilization. Eur Rev Med Pharmacol Sci. 2013;17:398-402.

Sfakianoudis K, Simopoulou M, Nitsos N, Rapani A, Pantou A, Vaxevanoglou T, et al. A Case Series on Platelet-Rich Plasma Revolutionary Management of Poor Responder Patients. Gynecol Obstet Invest. 2018;1-8.

van Eekelen R, Tjon-Kon-Fat RI, Bossuyt PMM, van Geloven N, Eijkemans MJC, Bensdorp AJ, et al. Natural conception rates in couples with unexplained or mild male subfertility scheduled for fertility treatment: a secondary analysis of a randomized controlled trial. Hum Reprod. 2018;33:919-23.

Veltman-Verhulst SM, Hughes E, Ayeleke RO, Cohlen BJ.. Intra-uterine insemination for unexplained subfertility. Cochrane Database Syst Rev. 2016;2:Cd001838.

Zadehmodarres S, Salehpour S, Saharkhiz N, Nazari L. Treatment of thin endometrium with autologous platelet-rich plasma: a pilot study. JBRA Assist Reprod. 2017;21:54-6. 